\title{
DETERMINANTS OF LARVAL MOLT INITIATION IN THE TOBACCO HORNWORM, MANDUCA SEXTA
}

\author{
LOUIS SAFRANEK AND CARROLL M. WILLIAMS
}

The Biological Laboratories, Harvard University. Cambridge, Massachusetts 02138

\begin{abstract}
Hornworm larvae adhere to Dyar's rule under normal growth conditions, increasing their live weight by an average of 5- to 6-fold from the outset of one instar to the next. This adherence to Dyar's rule is largely maintained even in instars subsequent to those in which larvae have been severely malnourished. The importance of relative weight gain for the normal onset of larval molting contrasts with the requirement for attainment of an absolute body weight prior to pupation. But body size is by no means the sole cue for the initiation of a larval molt. Thus, under conditions of malnutrition larvae can initiate a molt at any weight and can do so even in the absence of any weight gain in an instar: in these circumstances the duration of an instar is inversely related to the weight at the outset of malnutrition. In larvae fed ad lib as well as in malnourished larvae the initiation of a molt appears to be limited to a discrete phase of the photocycle. The failure of larvae to molt on schedule at body weights lower than those predicted by Dyar's rule cannot be attributed to an inability of the brain to stimulate ecdysone-dependent development as is the case in diapausing pupae. Though body size, instar duration, and photocycle all interact to determine the onset of the molt, the role of the brain remains ill-defined.
\end{abstract}

\section{INTRODUCTION}

Entomologist Harrison Dyar achieved a measure of lasting fame with his threepage 1890 paper reporting that the width of the head capsule of larval Lepidoptera increased from molt to molt by a factor that was generally constant for a given species-an empirical relationship that came to be known as "Dyar's law" or "Dyar's rule." Subsequent work suggested that every dimension of a larva progresses from one instar to the equivalent stage in the next by a constant factor, and so too does the larval weight (Bodenheimer, 1933). The rule was originally promulgated as a means for identifying successive instars, but its usefulness in this arena as well as its general validity have been repeatedly debated (for review, see Beck, 1950). Exceptions to the rule are abundant yet, beginning with Dyar's own observations, studies have repeatedly confirmed the applicability of the rule to at least some species under appropriate growth conditions. Well-documented examples include the cabbage looper Trichoplusia ni (Jones et al., 1981) and the tobacco hornworm Manduca sexta (Nijhout, 1975)-the latter the subject of the present paper.

Even in instances where Dyar's rule cannot be rigorously applied, a direct relationship between growth and the initiation of molting is typically apparent. But the actual mechanism by which larvae gauge their mass remains an enigma. Only in the two instances of the hemipterans Rhodnius prolixus and Oncopeltus fasciatus is an important relationship apparent between physical distention per se and the initiation

Received 30 May 1984; accepted 21 September 1984.

Abbreviations: LD, long day; SD, short day. 
of molting (Beckel and Friend, 1964; Blakley and Goodner, 1978; Nijhout, 1979)a link that may well be mediated by distention-induced activation of neural stretch receptors. In other instances including the tobacco hornworm our fragmentary understanding allows us to approach the functional circuitry only as a black box. To facilitate discussion we propose to name this unknown mechanism linking the attainment of a particular body size to the initiation of a molt the "Dyarstat."

Under normal feeding conditions the tobacco hornworm exhibits a remarkable adherence to Dyar's rule. This was first appreciated by Nijhout (1975) who showed that the live weight of the larval population increased between equivalent stages in successive instars by a factor of about 5.7 through the first four of the hornworm's five instars. Thus, the hornworm's Dyarstat can punctuate the typical course of larval exponential growth with molts at precise intervals. The present study focuses on the play of the Dyarstat under various feeding conditions and examines as well the contributions of growth, instar duration, and photocycle to the initiation of the molt. Though the Dyarstat exhibits a remarkable stability under many conditions, larval size proves to be only one of several factors that predictably shape the pattern of larval molting.

\section{MATERIALS AND METHODS}

Hornworms were reared at $25^{\circ} \mathrm{C}$ on an artificial diet as described by Truman (1972) and Bell and Joachim (1976) under either short-day (SD, 12L:12D) or longday (LD, 17L:7D) photoperiods. Under these conditions the larvae routinely underwent five larval instars. Time-of-day was arbitrarily referenced to lights-off at midnight $(24: 00=00: 00)$. The timing of events in the life cycle was as described by Truman (1972) and Truman and Riddiford (1974) except that we have termed the first $24 \mathrm{~h}$ of each instar Day 1 rather than Day 0.

In a feeding protocol described as the "steady-weight regimen" larvae were weighed and returned to individual, clean, capped plastic containers which contained no food. Thereafter at daily intervals each individual was weighed and provided with an amount of diet sufficient to restore it to near its original weight. This regimen was maintained until molting was initiated, as signaled by apolysis of the head capsule.

Removal of larval and pupal brains and implantation of brains into debrained diapausing pupae was accomplished as previously described (Safranek and Williams, 1980).

\section{RESULTS}

\section{Comparative development of first-and second-gate fourth-instar larvae}

In the experiments described here, groups of SD pharate fourth-instar larvae were selected from our stock at the outset of the photophase. All these larvae underwent ecdysis within the next $12 \mathrm{~h}$. We term this group of larvae beginning the fourth instar on the same day a cohort. Within a cohort the covert head-dependent events which initiate the molt to the fifth instar typically occur during the scotophase of either the third or fourth day of the instar (Truman, 1972). The overt initiation of the molt signaled by head capsule slipping ordinarily ensues half a day later during the photophase of the same day. In these experiments fourth instars of various ages were removed from their containers, weighed, and then returned to their food and observed for the onset of head capsule slipping. Larvae which underwent slipping on Day 3 of the instar were termed "first-gate larvae;" those which did so on Day 4, "secondgate larvae." 
In an examination of over a thousand pharate fourth-instar larvae studied in cohorts of 50-100 over the past several years, more than 95\% weighed 0.16 to 0.23 $\mathrm{g}$, the average ranging from 0.18 to $0.20 \mathrm{~g}$. First-gate and second-gate larvae were indistinguishable on the basis of their weights as pharate fourths; nevertheless larvae destined to be first-gate could be roughly separated from those of the second-gate by the hour of ecdysis to the fourth instar, the former tending to complete the molt earlier in the day by an average of several hours. By the outset of the photophase on the third day of the instar, weight could largely be relied on to distinguish these two groups. Thus, as illustrated in Figure 1, first-gate larvae appeared more frequently among the larger Day 3 larvae. Indeed, all individuals weighing at least $0.9 \mathrm{~g}$ initiated apolysis later on Day 3 whereas all but a few weighing $0.6 \mathrm{~g}$ or less waited until Day 4. Nearly twice as many first-gate as second-gate larvae were among the 246 individuals in this experiment; their average weights on the morning of Day 3 were $1.02 \pm 0.17$ $\mathrm{g}$ and $0.68 \pm 0.13 \mathrm{~g}$, respectively.

Although first-gate fourths are larger than their second-gate counterparts on the morning of Day 3, their final size at the time of apolysis to the fifth instar is typically less than that of second-gate larvae. The latter, having failed to initiate the molt during the gate on Day 3, are obliged to await the arrival of the gate on Day 4. As a result, the duration of the feeding period from ecdysis to the fourth instar until apolysis to the fifth instar is typically $15-18 \mathrm{~h}$ longer for second-gate than for firstgate larvae. This largely accounts for the 0.1 to $0.2 \mathrm{~g}$ average weight difference that may be seen between mature first- and second-gate fourths within a given cohort.

From data on the weight of several hundred larvae as pharate fourth and fifth instars we calculated a constant after the manner of Dyar-namely the quotient of the average weight at apolysis to the fifth instar divided by that at apolysis to the

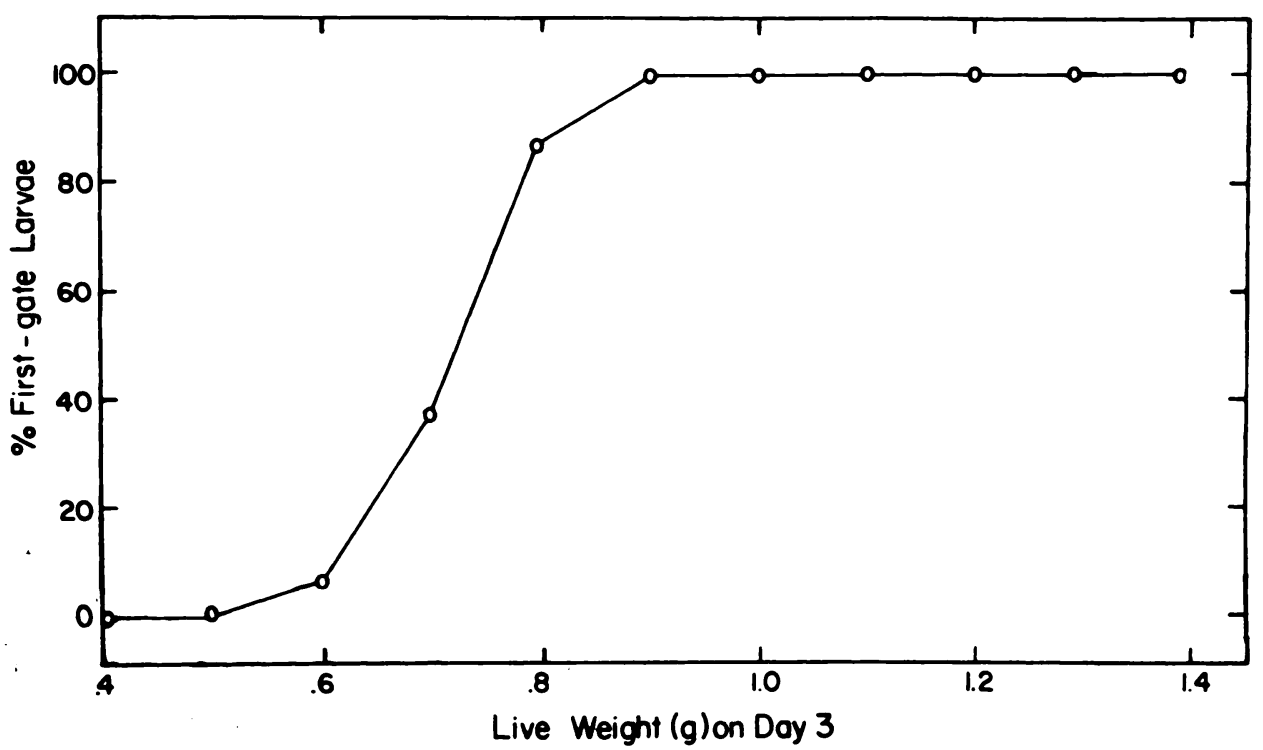

FIGURE 1. Weight-dependence of molt initiation by first-gate fourth-instar larvae. Fourth-instar SD larvae were weighed at the outset of the photophase (12:00) on Day 3 of the fourth instar and were considered first-gate larvae if head capsule slipping was apparent $24 \mathrm{~h}$ later. Each point represents the percentage of first-gate larvae in an interval extending $0.1 \mathrm{~g}$ above the corresponding weight on the $x$-axis. The number of larvae per category ranged from 5 to 10 at the extremes to $30-40$ over the range $0.6-1.0 \mathrm{~g}$. 
fourth. The calculated value of 5.8 was in remarkable agreement with the value of 5.7 found by Nijhout (1975). The individual values ranged from 4.4 to 8.4 , with larger values predominanting among second-gate larvae.

Effects of a steady-weight feeding regimen on the development of fourth-and fifth-instar larvae

SD fourth-instar larvae were divided into groups of 10-25 larvae at $0.1 \mathrm{~g}$ intervals between $0.15 \mathrm{~g}$ and $0.95 \mathrm{~g}$ and placed on a steady-weight regimen as described under Materials and Methods, daily receiving sufficient food to restore them to within .03 $\mathrm{g}$ of their initial weights. All survived to molt to the fifth instar except for 6 of 25 individuals placed on the regimen immediately after ecdysis to the fourth instar, these died without further development. Though many larvae molting at weights below $0.4 \mathrm{~g}$ failed to ecdyse, the old cuticle could readily be peeled away revealing in nearly all cases the presence of an intact fifth-instar larval cuticle. As shown in Figure 2, the duration of the instar was inversely related to the weight at the initiation of the steady-weight regimen. The instar was significantly prolonged for all groups of larvae

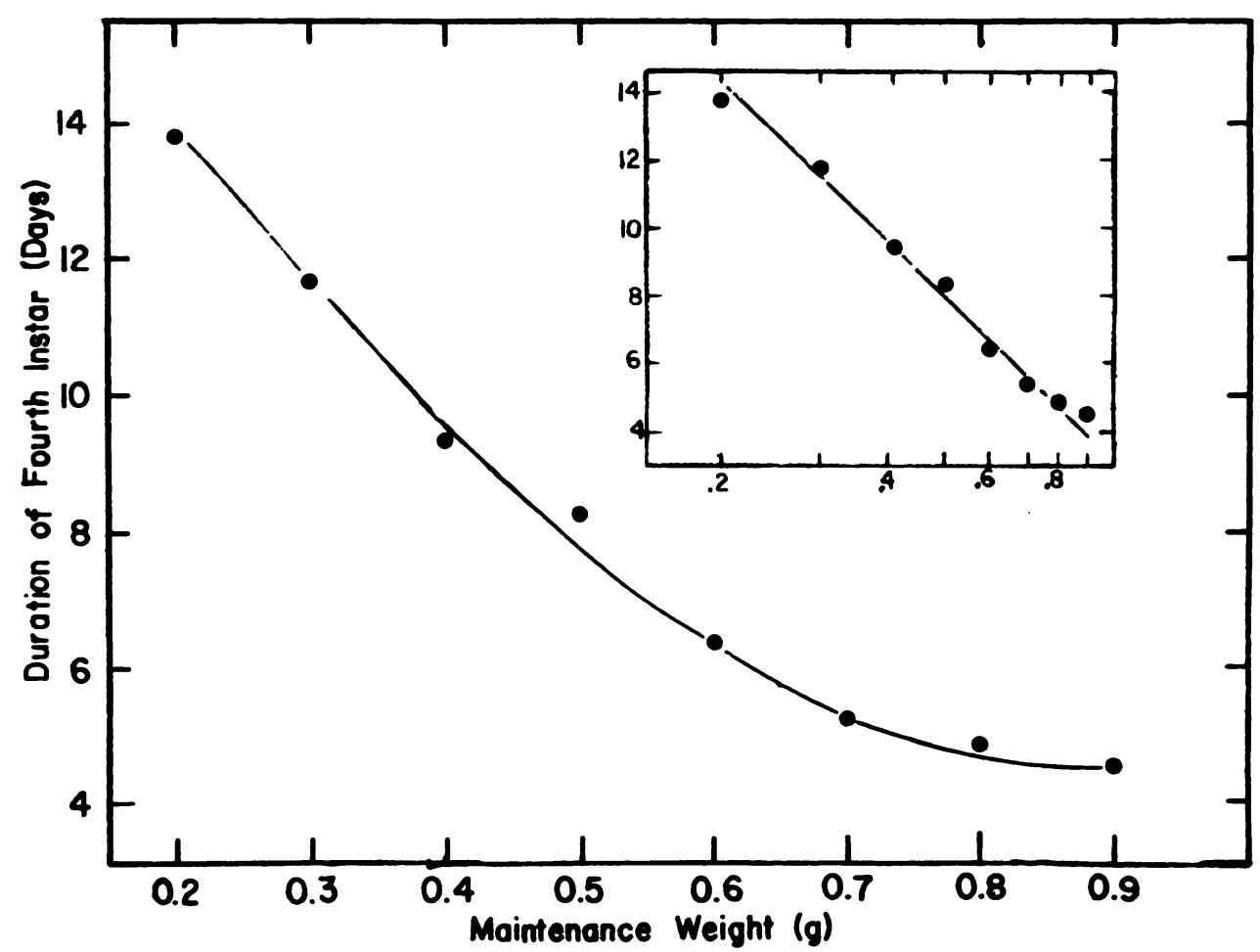

FIGURE 2. Inverse relationship between instar duration and the maintenance weight under a steadyweight regimen. Fourth-instar SD larvae weighing from 0.15 to $0.95 \mathrm{~g}$ were maintained on a steady-weight regimen as described under Materials and Methods and Results. The figure plots the duration of the instar from the time of apolysis to the fourth instar to the time of apolysis to the fifth instar as a function of the maintenance steady-weight. The standard deviation decreased steadily from 3 days at $0.2 \mathrm{~g}$ to 0 at $0.9 \mathrm{~g}$. Each point represents the average instar duration for 10 larvac except for the point at $0.2 \mathrm{~g}$ which represents 19 larvae. Each point represents an interval extending $\pm 0.05 \mathrm{~g}$ about the corresponding weight on the $x$-axis. The inset is a semilog plot of the same data. 
except those maintained at weights above 0.65 , most of which molted without a delay relative to similar larvae fed ad lib. When examined on a semilog plot, the data approximate a straight line.

A similar experiment was performed on 25 newly ecdysed SD fifth-instar larvae weighing from 0.8 to $1.2 \mathrm{~g}$ which were maintained by daily feedings within $0.05 \mathrm{~g}$ of their initial weights. In a fashion similar to the fourth instar, all individuals underwent apolysis, in this instance, after 16-23 days. They uniformly failed to ecdyse. Attempts to salvage several of them by manually removing the old cuticle proved to no avail since they failed to resume feeding. In every case a supernumerary sixth-instar larva had formed.

\section{Development of larvae after malnourishment in the third or fourth instars}

In the above experiments we found that under regimens of ad lib feeding larvae initiated the molt in a weight-dependent fashion after a weight increase of at least 4-fold; but under regimens of severe malnourishment they could molt after little or no weight gain within an instar. In additional experiments we examined the pattern of growth and molting in instars subsequent to those in which larvae had experienced severe malnutrition.

To this end, third-instar larvae weighing between 0.05 and $0.10 \mathrm{~g}$ were removed from diet and then maintained within this weight interval by small daily feedings; these weights correspond to about $25-50 \%$ of the maximum size typically attained by third instars fed ad lib. Larvae were weighed at the time of apolysis at the end of the third instar and daily thereafter. They were fed ad lib from the time of ecdysis to the fourth instar until the onset of the wandering period at the end of the fifth or sixth instar.

Figure 3 depicts the weight distribution of larvae at the outset of the fourth and fifth instars as well as the developmental fate at the end of the fifth instar for larvae from each $0.01 \mathrm{~g}$ division. As can be seen, larval weights were initially distributed more or less evenly. By contrast, larvae exhibited a bimodal distribution at apolysis to the fifth instar, by that time they had segregated into distinct weight categories which forecast their subsequent development. Thus, all larvae below $0.5 \mathrm{~g}$ underwent a supernumerary larval molt at the end of the fifth instar, while all those above this weight initiated metamorphosis. The two groups exhibited very different Dyar's ratios in the fourth instar, those with a subsequent supernumerary molt having an average ratio of 4.8, those metamorphosing at the end of the fifth instar an average ratio of 10.3. Though these two groups were separated by about $0.2 \mathrm{~g}$ at the end of the fourth instar, they became widely separated in weight prior to the onset of development at the end of the fifth instar: all fifths undergoing a supernumerary molt did so at weights below $2.5 \mathrm{~g}$, whereas those that underwent metamorphosis weighed over $6 \mathrm{~g}$ at the time of dorsal vessel exposure. The growth ratio from the fifth to the sixth instar for larvae undergoing a supernumerary molt averaged 4.3.

In a subsequent experiment we examined the development of SD larvae in the fifth instar after they had been subjected to a steady-weight regimen in the fourth instar at maintenance weights from 0.4 to $0.9 \mathrm{~g}$. In this instance larvae that were fed ad lib after molting to the fifth instar at weights above $0.6 \mathrm{~g}$ metamorphosed at the conclusion of the fifth instar at weights above $6 \mathrm{~g}$. Larvae fed ad lib after entering the fifth instar at 0.4 to $0.6 \mathrm{~g}$ either underwent metamorphosis as fifths at weights above $5 \mathrm{~g}$ or underwent a molt to supernumerary sixth instars at weights from 1.1 to $4.5 \mathrm{~g}$ (mean: $2.5 \mathrm{~g}$ ). Larvae undergoing a supernumerary molt exhibited an average Dyar's ratio of 5.2 . 


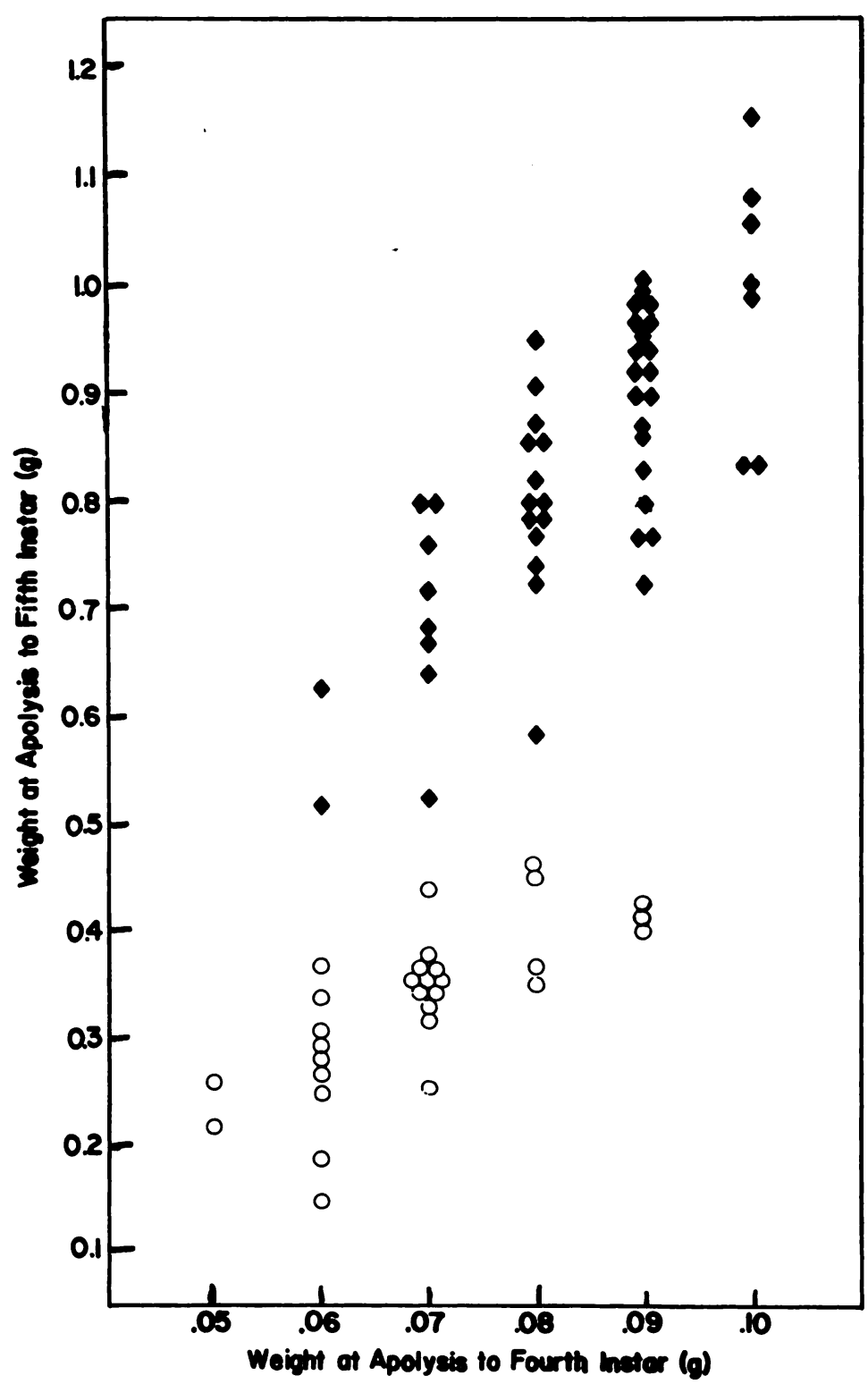

FIGURE 3. Weight of larvae at the time of apolysis to the fifth instar as a function of the weight at the time of apolysis to the fourth instar. Larvae were maintained on steady-weight regimens during the third-instar and fed ad lib thereafter. The duration of the fourth-instar for larvae initiating metamorphosis at the end of the fifth instar (solid diamonds) was typically one day longer than that for those molting to a supernumerary larval instar at the end of the fifth (clear circles). This accounts for their significantly greater proportionate weight increase in the fourth instar, but careful analysis of the growth data through the fourth instar fail to suggest a reason for this additional day of growth.

\section{Is molting by malnourished larvae gated by the photocycle?}

We inquired whether the initiation of larval molting at the conclusion of the fourth instar was coupled to the photocycle under conditions of malnutrition as it 
is under ad lib feeding (Truman, 1972). To this end we recorded the onset of head capsule apolysis in groups of normally fed first-gate $(n=129)$ and second-gate $(n$ $=250$ ) SD larvae and in a group of SD larvae maintained on a steady-weight regimen at weights between 0.4 and $0.5 \mathrm{~g}(\mathrm{n}=60)$. Figure 4 depicts the percentage of larvae initiating head-capsule slipping in different phases of the $24 \mathrm{~h}$ photocycle. Eighty

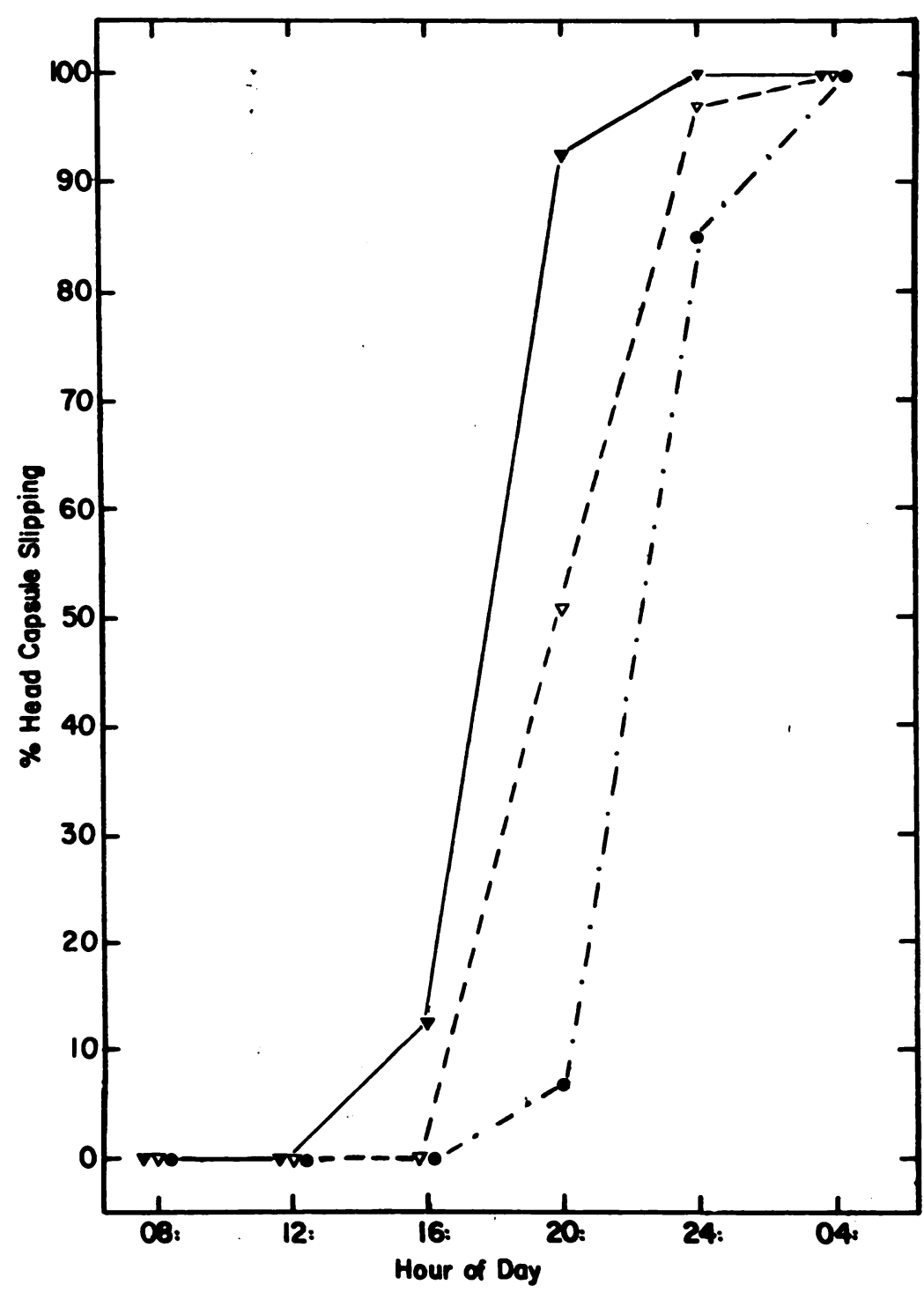

FIGURE 4. Cumulative percentages of first-gate (clear triangles), second-gate (dark triangles), and malnourished larvae (dark circles) initiating head capsule slipping at $4 \mathrm{~h}$ intervals at the end of the fourth instar. The day indicated on the abscissa extends from Day 3 to Day 4 of the fourth instar for first-gate larvae and from Day 4 to Day 5 for second-gate larvae. Malnourished larvae were observed at $4 \mathrm{~h}$ intervals during the several days over which they underwent apolysis: the data presented are derived by collapsing the observations of these several days into one. Larvae were observed under red light during the scotophase. The hour of the day was referenced to lights-off at midnight $(24: 00=00: 00)$. 
percent of second-gate larvae slipped their head capsules during the middle third of the photophase; first-gate larvae often did so during its final third. The majority of starved larvae also slipped their head capsules during the final third of the photophase, though some delayed until the ensuing scotophase. Thus, although the time of headcapsule slipping is shifted to the last portion of the photophase or slightly later, initiation of molting apparently remains closely coupled to the photocycle even in severely malnourished larvae.

\section{Assay of ecdysiotropic activity of larval brains}

At the outset of the fourth-instar larvae on an ad lib feeding regimen are 3-4 days from the initiation of the next molt. As we have seen, malnutrition early in this period can delay the onset of molting for as long as two weeks. We inquired as to whether the ecdysiotropic activity of the brain during the early fourth and fifth instars was curtailed as in the case of the diapausing pupal brain (Safranek and Williams, 1980). To this end we removed the brains from normally fed as well as malnourished larvae during the fourth or fifth instars. As further controls, we bioassayed brains excised from newly ecdysed diapause and non-diapausing pupae. The ecdysiotropic activity of each brain was assayed by implanting it into the head of a one-day-old diapause-destined pupa whose own brain had been simultaneously excised. The assay pupae were then examined daily for initiation of development as signaled by tracheal apolysis in the wings.

As summarized in Table I, brains from every group of larvae provoked the initiation of adult development by the assay pupae at similar rates and resembled in this regard the isolated brains of non-diapausing pupae. All were clearly more active than the brains of diapause-destined pupae. Manifestly, the larval brains even from malnourished larvae early in the fourth instar were not without significant ecdysiotropic

TABLE I

Ecdysiotropic activity of lanval hornworm brains in a pupal assay

\begin{tabular}{lc}
\hline \multicolumn{1}{c}{ Stage of donor } & Mean day of tracheal apolysis \\
\hline Fourth instar, Day 1 & 10 \\
Fourth instar, Day 2 & 9 \\
Fourth instar, Day 3 & 12 \\
Fourth instar, Day 4 & 10 \\
Fourth instar, Day 5 & 11 \\
Fifth instar, Day 1 & 11 \\
Fourth instar, steady-weight & 11 \\
LD pupa, Day 1 & 8 \\
SD pupa, Day 1 & 73 \\
\hline
\end{tabular}

Brains were removed from SD larvae or from LD or SD pupae at the several stages shown and were implanted singly into brainless diapause-destined pupac less than one-day-old. Assay pupace were examined daily thereafter for tracheal apolysis. The mean value is the average day on which tracheal apolysis occurred in each group of 10-12 assay pupae. Standard deviations ranged between one and two days for all groups except SD pupae which had a deviation of four weeks. The operations were performed during the last six hours of the photophase on the day indicated. Day 3 fourths were all second-eate larvae which would have initiated the molt during the ensuing scotophaxe on Day 4. Day 4 fourths were also second-gate larvac which had just initiated head capeale stippine Day 5 fourths were actually pharate fifth instars several hours prior to ecdysis. Steady-weight fourth instars were removed from diet at weights between .40 and .50 \& on Day 2 and placed on a steady-weight regimen: their brains were removed for astay on Day 4, several days prior to the time when similar unoperated larvae underwent apolysis. 
activity. These data suggest that the developmental delay witnessed in malnourished larvae is not due to the exercise of a diapause-like program by the brain.

\section{Discussion}

Exponential growth of hornworm larvae is punctuated by molts in a remarkably regular pattern: in each of the first four instars the average weight under normal conditions increases between molts by a constant factor. The constant of 5.7 described in Nijhout's detailed original study (Nijhout, 1975) continues very nearly to characterize our present laboratory stock, which exhibited a factor averaging between 5.5 and 6 in the present study of fourth-instar hornworms.

Within the constancy exhibited by the population as a whole, the Dyar's factor of individual fourth-instar hornworms varied considerably. This apparently stemmed largely from two sources. Because the initiation of molting is limited to a certain portion of the photocycle, larvae which attain a weight appropriate for molting continue to feed and grow until the permissive interval or "gate" arrives. In the case of fourthinstar larvae this period of growth may vary from 0 to at least $12 \mathrm{~h}$ and thus might have been responsible for weight differences of $0.25 \mathrm{~g}$ or more at the time of molting. A second source of variation is the approximately $18 \mathrm{~h}$ interval which routinely elapses from the point at which induction of the molt no longer requires the presence of the head until the onset of head capsule apolysis. In our experience this interval is of nearly constant duration regardless of whether larvae are feeding or being starved: consequently, any variations in the rate of growth at this time are not compensated by a change in the duration of feeding and so have significant effects on the ultimate size attained.

The present studies permit definition of a more reliable developmental threshold for larval molting. Small first-gate fourth-instar larvae on the morning of Day 3 are likely to be of a weight just adequate to have permitted the head-dependent events that initiate a molt to be completed during the gate on the preceding night. In the experiments here (Fig. 1) the smallest first-gate larvae at this time weighed $0.7-0.8$ $\mathrm{g}$-about 4 times as much as at the outset of the instar. In the further experiment summarized in Figure 2, only larvae weighing at least $0.7-0.8 \mathrm{~g}$ regularly initiated a molt without delay when further growth was prevented by starvation. Thus, once again acquisition of a weight about four times that at the outset of the instar appeared adequate for the initiation of molting at the ensuing gate. These experiments suggest that under conditions of ad lib feeding a four-fold increase in body weight prior to the end of the photocyclic gate is necessary for the completion of those head-centered events necessary for the induction of molting in the normal fourth instar.

When periods of malnutrition in the third or fourth instars forced larvae to molt at lower than normal weights, the Dyar's factors calculated for subsequent instars were generally between 4 and 5.5 . These were slightly lower than the population average of normal larvae but near the average of first-gate larvae. Whether the majority of these larvae were effectively first-gate larvae or whether in fact the Dyarstat had been reset to a slightly lower weight range we cannot say.

Although molt initiation was a function' of size under ad lib feeding conditions, under conditions of malnutrition molting could occur at any weight when sufficient time was allowed. Indeed, larvae in both the fourth and fifth instars proved able to initiate a molt in the absence of any increase from their weight at the time of ecdysis. Dyar (1890) suggested that the regular saltatory growth which he witnessed in the size of the head capsule could be used to identify successive larval instars. Although this system can be successfully applied to the hornworm under optimal feeding con- 
ditions, it cannot be generalized. The size of the head capsule is related to body size at the outset of the instar as Nijhout (1975) has clearly shown. Thus, under conditions of malnutrition, larvae can molt over a continuous weight range with a corresponding range of head capsule sizes. Manifestly under these conditions Dyar's rule can be used neither to predict the size at which larvae will undertake a molt nor to determine the larval instar. Consequently, Dyar's law is valid only under superior growth conditions.

Under the steady-weight regimen employed here, fourth instars exhibited an inverse relationship between instar duration and their maintenance weight. This variable dependence of molt initiation on body size may reflect different mechanisms at play; for example, the Dyarstat may be employed under conditions of ad lib feeding, whereas another mechanism, perhaps the mere passage of time, may accomplish under conditions of malnourishment what is normally mediated via the Dyarstat. Alternatively, the setting on the Dyarstat may be variable rather than absolute: in this case, the weight threshold for initiating a molt might be lowered slightly each day until it matches the actual weight of the malnourished larva. Whatever the mechanism, starvation clearly short-circuits the normal link between attainment of a particular size and the initiation of ecdysone-dependent development. This appeared to be a general effect of malnourishment in these studies since larvae from the third, fourth, and fifth instars, when underfed, regularly initiated development at a fraction of their typical mature weights.

In the accompanying paper (Safranek and Williams, 1984) we demonstrate that malnourished fifth-instar larvae are able to initiate metamorphosis only if they have attained a weight of at least about $3 \mathrm{~g}$. This development threshold differs fundamentally from the weight thresholds for larval molting that we describe here. Thus, whereas larval molting may occur at virtually any weight in at least the late third, fourth, and early fifth instars, initiation of metamorphosis absolutely required attainment of a weight beyond the interval of 2 to $3 \mathrm{~g}$. Moreover, whereas under conditions of ad lib feeding the initiation of larval molting requires a several-fold increase in body size within an instar, the initiation of metamorphosis in the final instar depends only on the attainment of a body size of 2-3 g regardless of the fold increase in body weight. Thus, larvae manifest abilities to gauge their size both relative to earlier points in the instar or life history as well as on an absolute scale.

Under conditions of ad lib feeding the initiation of molting normally occurs in strict relation to the photocycle after the attainment of an appropriate weight. And though under adverse feeding conditions molting was initiated at much lower weights, even under these conditions the initiation of molting appeared to be closely tied to the photocycle. Apparently when molting occurs under conditions of severe malnourishment it continues to be properly orchestrated and does not represent merely the breakdown of normal control mechanisms.

Assays of PTTH-activity in the brains of young fourth-instar larvae under conditions both of ad lib feeding and of malnutrition revealed levels of activity clearly greater than those exhibited by the diapausing pupal brain and similar to those of non-diapausing pupal brains as well as of brains from mature fourths about to initiate a molt. These findings suggest that the failure of young intermolt larvae or of malnourished larvae to initiate a molt is not due to a diapause-like condition of the brain at these periods.

In the absence of adequate data detailing the pattern of hemolymph PTTH titers in hornworm larvae, these experiments raise the possibility that our present models describing the regulation of larval molting are incomplete. Certainly the requirement for a brain is absolute, since even mature fourth-instar larvae deprived of their brains 
just before molt initiation uniformly fail to undertake any subsequent development, although they may live for over two weeks (Safranek and Williams, 1980). But whether a distinct change in the PTTH output of the brain occurs at the outset of a molt as is classically envisioned is by no means certain. Certainly a significant body of observations in the literature documents that some insects can generate a molt-inducing level of ecdysone in the absence of the brain, among these the hornworm itself from the final instar through the subsequent metamorphic molts (Safranek and Williams, 1980). Manifestly PTTH-independent mechanisms for driving a molt exist, and these may well prove to be critically involved even in molts such as the hornworm's larvallarval molts where the presence of the brain is indispensable. Though some mechanism must operate near the outset of a larval molt to integrate information concerning larval size, duration of the instar, and phase of the photocyle, it is by no means clear whether the brain plays the role of maestro or of metronome.

\section{ACKNOWLEDGMENTS}

This work was generously supported by funds from the N.I.H. and the Rohm and Haas Company. We gratefully acknowledge the patient and dedicated assistance of a host of Harvard College undergraduates in the execution of the various feeding regimens herein described.

\section{LITERATURE CITED}

BECK, S. D. 1950. Nutrition of the European corn borer Pyrausta nubilalis (Hbn.). II. Some effects of diet on larval growth characteristics. Physiol. Zool. 23: 353-361.

BECKEL, W. E., AND W. G. FrIEND. 1964. The relation of abdominal distention and nutrition to molting in Rhodnius prolixus (Stahl) (Hemiptera). Can. J. Zool. 42: 71-78.

BELL, R. A., AND F. G. JOACHIM. 1976. Techniques for rearing laboratory colonies of tobacco hornworms and pink bollworms. Ann. Entomol. Soc. Am. 69: 365-373.

BLAKLEY, N., AND S. R. GOODNER. 1978. Size-dependent timing of metamorphosis in milkweed bugs (Oncopeltus) and its life history implications. Biol. Bull. 55: 499-510.

BODENHEIMER, F. S. 1933. The progression factor in insect growth. Q. Rev. Biol. 8: 92-95.

DYAR, H. G. 1890. The number of molts of lepidopterous larvae. Psyche 5: 420-422.

JONES, D., G. JONES, AND B. D. HAMMOCK. 1981. Growth parameters associated with endocrine events in larval Trichoplusia ni (Hubner) and timing of these events with developmental markers. $J$. Insect Physiol. 27: 779-788.

NuHout, H. F. 1975. A threshold size for metamorphosis in the tobacco hornworm, Mandrica sexta (L.). Biol. Bull. 149: 214-225.

NuHout, H. F. 1979. Stretch-induced moulting in Oncopeltus fasciatus. J. Insect Physiol. 25: 277-281.

SAFrANEK, L., AND C. M. Williams. 1980. Studies of the prothoracicotropic hormone in the tobacco hornworm, Manduca sexta. Biol. Bull. 158: 141-153.

SAFraneK, L., AND C. M. WilliamS. 1984. Critical weights for metamorphosis in the tobacco hornworm, Manduca sexta. Biol. Bull. 167: 555-567.

Truman, J. W. 1972. Physiology of insect rhythms. I. Circadian organization of the endocrine events underlying the moulting cycle of larval tobacco hornworms. J. Exp. Biol. 57: 805-820.

TRUMAN, J. W., AND L. M. RIDDIFORD. 1974. Physiology of insect rhythms. III. The temporal organization of the endocrine events underlying pupation of the tobacco hornworm. J. Exp. Biol. 60: 371382. 